Research Article

\title{
Prediction Method of College Students' Psychological Pressure Based on Deep Neural Network
}

\author{
Bing Wang and Sitong Liu \\ School of Traffic and Transportation, Northeast Forestry University, Haerbin 150000, China \\ Correspondence should be addressed to Sitong Liu; liusitong@nefu.edu.cn
}

Received 9 October 2021; Revised 26 October 2021; Accepted 3 November 2021; Published 12 November 2021

Academic Editor: M Pallikonda Rajasekaran

Copyright ( 2021 Bing Wang and Sitong Liu. This is an open access article distributed under the Creative Commons Attribution License, which permits unrestricted use, distribution, and reproduction in any medium, provided the original work is properly cited.

\begin{abstract}
Aiming at the problems of low prediction accuracy and efficiency and poor prediction effect in the current psychological pressure prediction methods, a psychological pressure prediction method for college students based on deep neural network is proposed. The structure and algorithm of depth neural network and gray theory model are analyzed. Using the deep neural network, this paper establishes the sample set data of college students' psychological pressure prediction and constructs the college students' psychological pressure prediction model combined with the deep neural network algorithm of gray theory. The physical network information model is formed through the relationship between neurons. According to the dynamic changes of college students' psychological pressure in each neuron of the physical network, the prediction of college students' psychological pressure is completed. The experimental results show that the proposed method is effective in predicting college students' psychological pressure and can effectively improve the accuracy and efficiency of college students' psychological pressure prediction.
\end{abstract}

\section{Introduction}

Psychological pressure is a state of mental and physical tension that individuals tend to show through various psychological and physiological reactions when they perceive or recognize that they are facing the important and difficult environmental requirements [1-3]. High-efficiency life has inevitably brought people a sense of urgency and pressure, and the mental health problems of college students have become increasingly prominent $[4,5]$. Due to a series of problems, such as academic, interpersonal, emotional, and job competition, college students are prone to negative emotions, such as inferiority, jealousy, depression, irritability, anxiety and fear, and even violent tendencies, which will cause serious harm to themselves and even society in the long run. College students are a special social group. They carry the expectations of society and family and have a strong desire to become talented. However, because their psychology is undergoing a transition period from juvenile immaturity to adult maturity, their mind are still immature and their adaptability is poor [6,7]. During this period of time, various pressures are particularly easy to form psychological problems. Therefore, it is of great significance to predict the psychological stress of college students.

At present, scholars in related fields have studied the prediction of psychological pressure. Lima et al. [8] proposed a prediction method of temperament and psychological types based on twitter data. This method briefly reviews the temperament theory and analyzes social media data based on it, so as to achieve the purpose of predicting temperament and psychological types. Subsequently, a framework was proposed to predict temperament and psychological types through linguistic and behavioral analysis of Twitter data. Infer the temperament type according to David Keirsey's model, and infer the mental type according to the MBTI model. This method can effectively predict temperament and psychological type. Alharthi [9] proposed a mobile based context aware acute stress prediction method. Predict the user's stress state according to the user's current context data. The ECG signal is used to identify the user's pressure state. With the help of machine learning algorithms and cloud computing services, build an adaptive and personalized prediction model for user 
context generation. This method can effectively adapt to the user's stress state and the changing characteristics of the surrounding environment. However, the above methods still have the problems of low prediction accuracy and efficiency and poor prediction effect.

Aiming at the above problems, a prediction method of College Students' psychological stress based on deep neural network is proposed. Deep neural network has high-dimensional data processing ability and strong fault tolerance. After some neurons are damaged, it has little impact on the global training results. Therefore, this paper uses the deep neural network to establish the sample set data of College Students' psychological stress prediction and constructs the college students' psychological stress prediction model combined with the gray theory model. Through the interconnection between neurons, a physical network information model is formed. According to the dynamic changes of the psychological pressure of college students in each neuron of the physical network, the prediction of the psychological pressure of college students is realized. The prediction effect of this method is good, and it can effectively improve the prediction accuracy and efficiency.

\section{Depth Neural Network}

As the main implementation technology of deep learning, deep neural network (DNN) occupies an absolute position in deep learning. Deep neural network also includes artificial neural network, convolutional neural network, and other different network structures [10-12].

2.1. Artificial Neural Network. Artificial neural network (ANN) is to extract some basic features of the nervous system in which the biological brain reacts to things and simulate this abstract process by program. By simulating the response mechanism of the brain, the neural network is the process of processing and analyzing the received data and handing the processing results to the effector to make some command [13]. The artificial neural network is adaptive, and its most basic component is neurons. The neuron structure is shown in Figure 1.

In Figure $1, x_{n}$ is the input of the neuron, $w_{n}$ is the connection weight of the input element and the unit, $a$ is the threshold of the neuron, $h$ is the activation function, and $o$ is the output of the generated neuron, expressed as

$$
o=h\left(\sum_{i=1}^{n} x_{i} w_{i}+b\right) .
$$

In formula (1), $b$ is the bias term of the neuron [14].

(1) There is a group of connections, and the weight of each connection is represented by the weight. When the weight is greater than zero, the connection is opened, and when it is less than zero, the connection is closed.

(2) It has summation function to calculate the weighted sum of input signals.

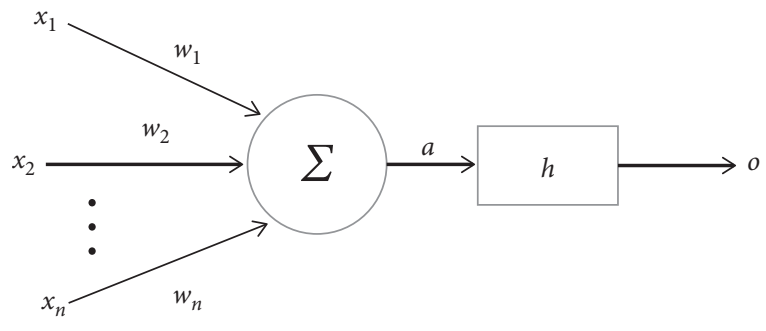

FIGURE 1: Neuron structure.

(3) Nonlinear activation function is as follows: common activation functions $\varphi(\cdot)$ include Sigmoid function, piecewise linear function, and threshold function. There is also a threshold $\theta$ (or bias $-\theta$ ).

In addition to the characteristics of neural nodes, artificial neural networks also have the characteristics of topology. There are two main connection modes:

(1) Feedforward network: each neuron in the network layer only accepts the input data of the previous layer and then outputs it to the nodes of the next layer. There is no feedback in the middle.

(2) Feedback network: all nodes in the network are computing units and can also receive data input and output data to the outside world. Each connecting arc can flow in both directions.

Feedforward network is mainly used for function approximation and pattern recognition. There are two kinds of feedback networks according to the utilization of the minimum points of the energy function. The first kind is that all the minimum points of the energy function have their functions, and this kind is mainly used as various memories. The second kind only uses the global minimum, which is mainly used to solve the optimization problem.

Artificial neural network also has three characteristics:

(1) Automatic learning: it is expected that the future artificial neural network computer will provide economic prediction, market prediction, and benefit prediction for mankind, and its application prospect is very broad.

(2) Associative storage: the feedback network has the ability of associative storage.

(3) It can find the optimal solution at high speed: the optimal solution of complex problems needs a lot of computation. The feedback neural network model can use the powerful computing power of the computer to find the optimal solution.

2.2. Convolutional Neural Network. Convolutional neural network $(\mathrm{CNN})$ is a differentiated depth model. It is the first successfully trained depth neural network algorithm in multilayer network structure. CNN is a deep neural network framework based on minimizing the requirements of preprocessing data. Affected by the early delayed neural network, it uses weight sharing in time dimension to reduce the 
complexity of training [15-17]. The structure of convolutional neural network is shown in Figure 2.

Due to the unique convolution structure of the network, it can compress the input data to the greatest extent without losing the original data features. The structure and training method of convolutional neural network is described to provide a theoretical basis for the subsequent prediction model. Convolutional neural network is mainly divided into the following three parts.

(1) Convolution layer: it is the core structure of convolution neural network, and most calculations are concentrated in convolution layer $[18,19]$. It is assumed that the position of the moving real object at time $t$ is $x(t)$. The smaller the time interval between the real object and a certain position, the higher the correlation between the measurement results, and there is a proportional relationship between them. This relationship is expressed by the weighting function $w(a)$, and $a$ represents the time interval. Then, the expression of the continuous estimation function $S$ of the position of the moving object can be obtained as follows:

$$
S(t)=\int x(a) w(t-a) d a .
$$

In general, a specific symbol $\otimes$ is used to represent the convolution operation, and the function expression after using the specific symbol is

$$
S(t)=(x \otimes w)(t) .
$$

In formula (3), $S(t)$ is the feature relationship mapping, $x$ is the input, and $w$ is the kernel function. When the time variable in formula (3) is taken as an integer, the cumulative addition expression for discrete volumes is written as follows:

$$
S(t)=(x \otimes w)(t)=\sum_{n=-\infty}^{\infty} x(a) w(t-a) .
$$

(2) Excitation layer: in general, the excitation function used in the excitation layer uses a nonlinear function, and its main purpose is to solve the linear problem of the neural network, so as to overcome the problem that the general neural network cannot solve the nonlinear problem [20]. The activation function of the convolutional neural network has two forms: the Sigmoid function and the tanh function of its deformable body. The definition of the Sigmoid function is as follows:

$$
g(Z)=\frac{1}{1+e^{-z}}
$$

The Sigmoid activation function curve is shown in Figure 3.

According to formula (5), it can be seen that the function of the Sigmoid function is to transform a real number input to an output between 0 and 1 .
However, in actual use, due to the division operation in the Sigmoid function, the speed of its derivation becomes very slow. When the Sigmoid function is close to the two endpoints of 1 and 0 , its gradient is almost close to 0 , making the model unable to update the parameters. There is a phenomenon of disappearance of the gradient [21]. In order to overcome the shortcomings of the above-mentioned Sigmoid function, the Sigmoid function is modified and improved, and the tanh activation function is obtained. The mathematical definition of the tanh activation function is

$$
\tanh (x)=\frac{e^{x}-e^{-x}}{e^{x}+e^{-x}}
$$

The tanh function solves the slow convergence problem of the Sigmoid function to a certain extent, but the problem of its gradient disappearance is still inevitable, but overall, the performance of the tanh function has been better than the Sigmoid function [22]. The tanh activation function curve is shown in Figure 4.

(3) Pooling layer: it is a particularly important layer structure in the convolutional neural network. Its main function is to gradually reduce the size of the data volume, thereby reducing the amount of parameters in the network and reducing the waste of computing resources. At the same time, the effect of compressing the size of the data can also reduce the occurrence of overfitting to a certain extent and improve the robustness of features [23]. The processing process of the maximum pooling layer is shown in Figure 5.

At present, there are two types of pooling layers commonly used: average pooling (AP) and maximum pooling (MP). The average pooling layer outputs the average result and the maximum pooling outputs the maximum result.

Common convolution neural network forms mainly put some convolution layers and activation function layers together, add some standardization layers in front of the activation function layer, and then follow the pool layer. After that, repeat until the input data is compressed to a small enough size, then expand the feature map, connect the full connection layer, and finally output the results [24-26].

2.3. Gray Theory Model. Gray theory mainly studies small behavior samples with some known information and takes uncertain systems as the research object. Through the formation, development, and extraction of known information, the correct understanding and effective control of the system can be realized $[27,28]$. The basic principle is to form a series of exponential functions classified by the accumulation of positive data, then generate the function through the sequence, and finally generate the final sequence through the solution of the differential equation.

The modeling of gray theory is divided into five stages: language model, network model, quantitative model, 

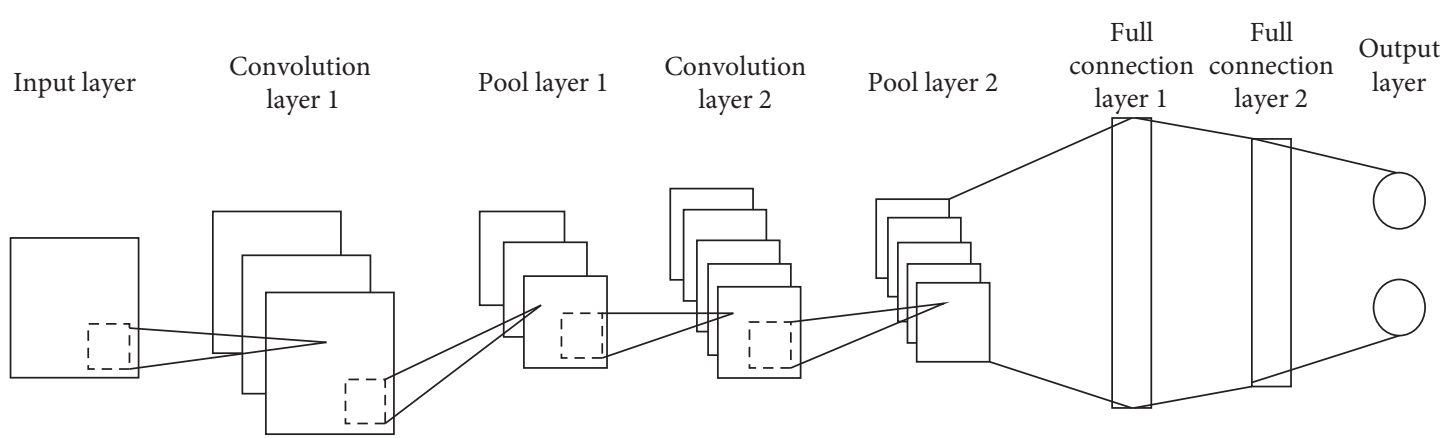

FIGURE 2: Convolutional neural network structure.

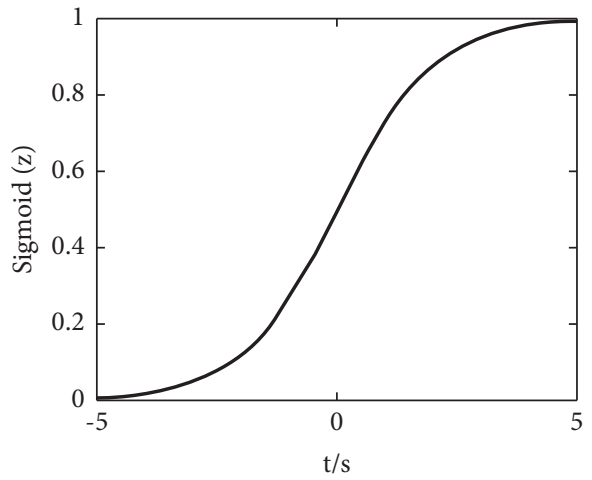

FIGURE 3: Sigmoid activation function curve.

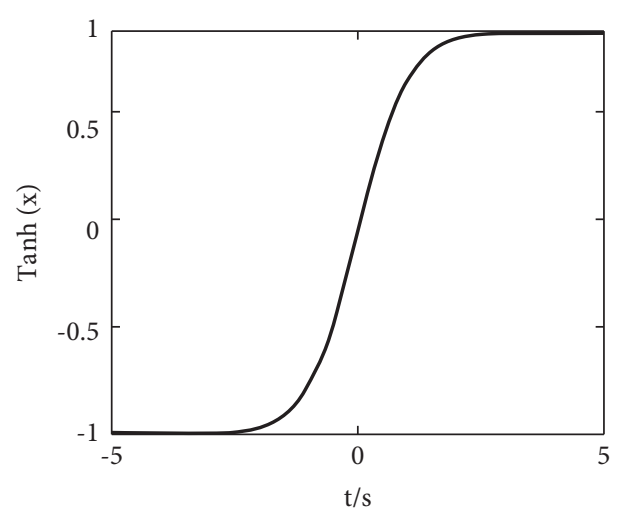

FIgURE 4: Tanh activation function curve.

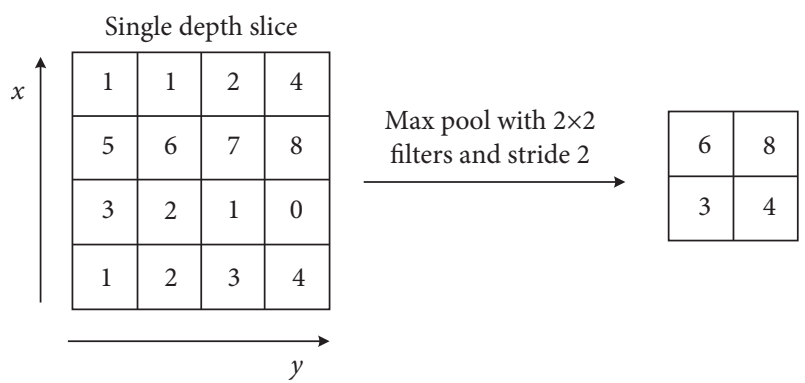

FIGURE 5: Processing process of maximum pool layer. dynamic model, and optimization model [29]. The gray system model is a system with incomplete information. Generally, the gray system can use $k$ variables and $n$ order differential equation models $G M(n, k), n$, and $k$ to represent different meanings. In gray theory, the most commonly used model is the standard gray model. The modeling process is as follows. First, add the original data to calculate

$$
x^{(0)}=\left(x^{(0)}(1), x^{(0)}(2), \ldots, x^{(0)}(n)\right) \text {. }
$$

Among them, $x^{(0)}$ is the initial value and $n$ is the sequence variable. Perform the second modeling sequence based on the original data results:

$$
x^{(1)}=\left(x^{(1)}(1), x^{(1)}(2), \ldots, x^{(1)}(n)\right) \text {. }
$$

Then, the whitening differential equation is established:

$$
\frac{\mathrm{d} x^{(1)}}{\mathrm{d} t}+f x^{(1)}(t)=l .
$$

In formula (9), $f$ is the development coefficient and $l$ is the gray-scale effect. Then, calculate the parameter vector $U=[f, l]^{T}$. Estimate the parameters $f$ and $l$ using the least square method. The estimated value of $U$ that reaches the minimum value is

$$
U=[f, l]^{T}=\left(B^{T} B\right)^{-1} B^{T} Y .
$$

By solving the previous equation,

$$
\widehat{x}(k+1)=\left(x^{(0)}(1)-\frac{\widehat{l}}{\widehat{f}}\right) e^{-\widehat{a} k \frac{\widehat{l}}{\widehat{f}}}, k=0,1, \ldots, n-1 .
$$

Finally, the previous predicted value is $\widehat{x}(k+1)$ and the model recovery value is $\widehat{x}^{(0)}(k+1) . \widehat{x}^{(0)}(k+1)$ is the gray model obtained.

\section{Prediction Methods of College Students' Psychological Pressure}

This paper applies deep neural network to college students' psychological pressure prediction, establishes the sample set data of college students' psychological pressure prediction, and realizes the establishment of college students' psychological pressure prediction model. The physical network information model is formed through the relationship between neurons. According to the dynamic changes of college 
students' psychological pressure in each neuron of the physical network, the prediction of college students' psychological pressure is completed.

\subsection{Establishing the Sample Set Data of College Students'} Psychological Pressure Prediction. In order to better predict college students' psychological pressure, firstly, the deep neural network [30] is used to establish the sample set data of college students' psychological pressure prediction. The specific operation steps are as follows.

Assuming that the psychological stressors and psychological pressure response of college students are $X_{i}$ and $X_{j}$, respectively, in the two time periods of $i$ and $j$, the relationship between $X_{i}$ and $X_{j}$ is calculated as $W_{\left\{X_{i}, X_{j}\right\}}$, and the set of college students' psychological pressure behavior is $d_{\text {wergv }}^{\prime \prime}$; then the physical network information model is expressed as follows:

$$
S_{\mathrm{SFG}}^{\prime}=\frac{d_{\mathrm{wergv}}^{\prime \prime}+W_{\left\{X_{i}, X_{j}\right\}}}{\left\{X_{i}, X_{j}\right\}}+\frac{\{i, j\}}{j_{\mathrm{jkl}}{ }^{\prime}} .
$$

In formula (12), $j_{\mathrm{jkl}}^{\prime}$ is the weight of the effective psychological pressure behavior of college students.

Assuming that the random variable of the psychological pressure behavior of college students is $r_{\text {wert }}^{\prime}$, the deep neural network prediction node of the $d_{\text {wergv }}^{\prime \prime}$ behavior set is $Q_{\text {aqwd }}^{\prime}$, and $p_{a}\left(X_{i}\right)$ represents the function set of $X_{i}$ deep neural network prediction nodes; then, the deep neural network predicts the psychological pressure behavior of college students as

$$
D_{\mathrm{DF}}^{\prime}=d_{\text {wergv }}^{\prime \prime} \times \frac{p_{a}\left(X_{i}\right)-Q_{\mathrm{aqwd}}^{\prime}}{r_{\text {wert }}^{\prime} \times p_{\text {werkk }}^{\prime} \times X}-d_{\mathrm{dfg}}^{\prime} .
$$

In formula (13), $p_{\text {werkk }}^{\prime}$ is the distribution feature of each deep neural network node $X_{i}$, and $d_{\mathrm{dfg}}^{\prime}$ is the probability distribution of the set $X$ of predictive variables of psychological pressure for college students.

Assuming that the weight of the relationship between the psychological pressure and the psychological pressure response of college students is $\eta_{\text {sd }}^{\prime}$, and the probability of the psychological pressure behavior of college students is $s_{\text {sde }}{ }^{\prime}$, the establishment of the psychological pressure prediction sample set data of college students is expressed as

$$
S_{\mathrm{swdkk}}^{\prime}=\frac{s_{\mathrm{sde}}^{\prime} \times D_{\mathrm{DF}}^{\prime}}{\eta_{\mathrm{sd}}^{\prime}} S_{\mathrm{SFG}}^{\prime}-\frac{\left\{v_{\mathrm{fhj}}^{\prime}-h_{\mathrm{hjk}}^{\prime}\right\}}{k_{\mathrm{kl}}^{\prime}} .
$$

In formula (14), $v_{\text {fhj }}^{\prime}$ is the probability distribution of the deep neural network predicting the psychological pressure behavior of college students over time, $h_{\mathrm{hjk}}^{\prime}$ is the transfer function of the deep neural network predicting the psychological pressure of college students, and $k_{\mathrm{kl}}^{\prime}$ is the weight of the deep neural network predicting the psychological pressure of college students.

It can be seen from the above description that the deep neural network is used to predict college students' psychological pressure behavior and establish the sample set data of college students' psychological pressure prediction.

\subsection{Constructing the Prediction Model of College Students'} Psychological Pressure. In order to construct the prediction model of college students' psychological pressure, the depth neural network algorithm of gray theory is adopted, and the gray depth neural network is set as $[31,32]$

$$
N=\sum_{i}^{n}=X_{i}^{\prime} \omega
$$

In formula (15), $X_{i}^{\prime}$ is the $i$ input layer of the gray deep neural network.

Assuming $\omega x_{i}^{\prime} x_{j}^{\prime}$ is the psychological pressure prediction weight of the input layer of the deep neural network, the following formula is obtained:

$$
N_{j}^{\prime}=\sum_{i}^{n}=X_{i}^{\prime} \omega x_{i}^{\prime} x_{j}^{\prime} .
$$

Suppose $i$ psychological pressure of the gray deep neural network is $\mu x_{j}^{\prime} k$ and $\omega x_{j}^{\prime} k$ is the psychological pressure weight of the hidden layer; then, there is

$$
\begin{aligned}
& \mu x_{j}^{\prime} k=\sum_{i}^{n}=\mu X_{i}^{\prime} \omega x_{j}^{\prime} k, \\
& N x_{j}^{\prime}=\sum_{i}^{n}=\mu X_{j}^{\prime} \omega x_{j}^{\prime} k .
\end{aligned}
$$

Using the iterative calculation of formulas (15)-(18), the established prediction model of college students' psychological pressure based on deep neural network is simulated and trained.

Considering the dynamic changes of students' psychological pressure, the ECG waveforms of a subject under low pressure and high pressure are collected, as shown in Figure 6.

Based on the above analysis, in the deep neural network structure, a single neuron basically does not affect the judgment ability of the deep neural network. The prediction of college students' psychological pressure is the result of the cooperation of the deep neural network $[33,34]$. When a small part of neurons fails in the deep neural network, it will not have a great impact on the prediction results of college students' psychological pressure. Scattered information is gathered into a whole through neurons.

The deep neural network is used to model the psychological pressure prediction of college students, and the adaptive feature and deep neural network theory [35] are used to construct the psychological pressure prediction model of college students. In the input layer of the deep neural network, $k$ neurons can constitute the self-adaptive characteristics of the psychological pressure of college students, and for the output layer, $M$ neurons constitute the predictive value characteristics of the psychological pressure 


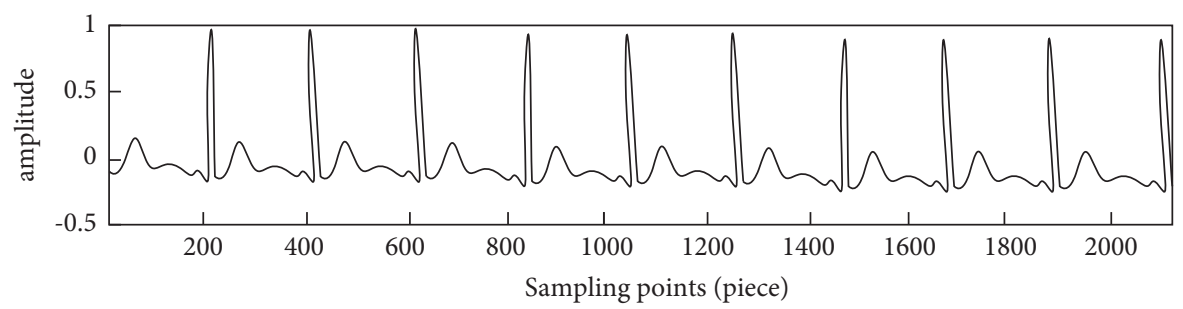

(a)

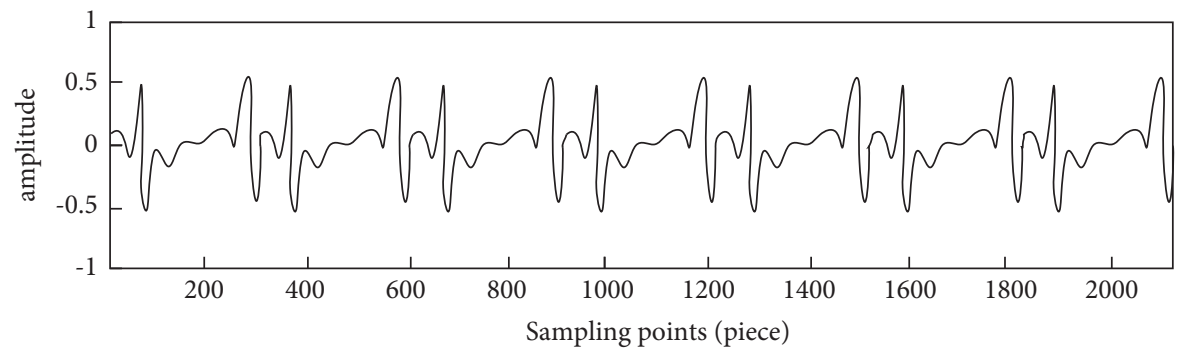

(b)

FIGURE 6: ECG waveforms under low voltage and high voltage. (a) Low-voltage ECG waveform. (b) High-voltage ECG waveform.

of college students. If the weight between the input layer node and the output layer node in the deep neural network is $W_{i j}$, where $i \in[1, k], j \in[1, m]$, the specific algorithm is as follows.

First, initialize the weight information $W_{i j}$ of the psychological pressure of college students and obtain the weight training set as $\left(x_{1}^{l}, x_{2}^{l}, \ldots, x_{h}^{l}\right), l \in[1, N]$.

Then, set the time to $t$ and obtain the vector $X_{i}^{l}(t)$ from the training set of the deep neural network. Then, calculate the Euclidean distance between each node in the deep neural network:

$$
d_{j}=\sum_{i=1}^{K}\left(X_{i}^{l}(t)-W_{i j}(t)\right)^{2} .
$$

Finally, by selecting the neurons in the deep neural network, combining the Euclidean distance $d_{j}$ obtained previously, the required neuron information is obtained, and the weight information is obtained by adjusting the two adjacent deep neural network nodes $N_{g}(t)$ :

$$
W_{i j}(t+1)=W_{i j}(t)+\eta(t)\left(X_{i}^{l}(t)-W_{i j}(t)\right) .
$$

In formula (20), $t$ represents the number of iterations of college students' psychological pressure prediction, and $\eta(t)$ represents the efficiency of training. Through Sigmoid, the psychological pressure prediction model of college students is activated by function [36], and the logarithm of Sigmoid is

$$
f(n)=\frac{1}{1+e^{-n}}
$$

In formula (21), $e$ represents the maximum coefficient in the deep neural network's psychological pressure prediction model for college students, and $n$ represents the number of all neuron nodes in the deep neural network, which can transform the prediction of college students' psychological pressure into an output signal $[-\infty,+\infty]$. When the numerical value of the psychological pressure prediction of college students is smaller, it means that the coefficient obtained is larger, so as to distinguish the input signal and input signal of the psychological pressure prediction model of college students and realize the prediction of the psychological pressure of college students.

\section{Experimental Analysis}

4.1. Experimental Environment and Dataset. In order to verify the effectiveness of the method for predicting college students' psychological pressure based on deep neural networks, a predictive model of college students' psychological pressure was established through the MATLAB platform, and the data was applied and analyzed. Using the Nntraintool generated by the MATLAB program deep neural network toolbox, call the dividevec() function in MATLAB to break the original order of the data, randomly divide the data into two categories, and randomly extract part of the data for network simulation.

Taking the psychological stress data of college students as the sample, this paper divides the effective data of college students' psychological stress prediction through formula (14) and uses $10 \%$ of them as the test sample for the simulation test of the prediction model and the remaining $90 \%$ for training the deep neural network model. Before training, set the network training parameters. After many experiments, finally set the network training set sample to 1000; the training target is set to $1 e-6$, and the learning rate is 0.01 . There are 100 test samples of the simulation prediction model. Use the load function to read the sample data, that is, load the data that has been entered and stored, and normalize the input data. Select the function mapminmax function. After the data are normalized, they are all in the interval $[1,-1]$ to realize the psychological pressure prediction of college students. 
In order to further verify the effectiveness of college students' psychological stress prediction method based on deep neural network, [8] temperament and psychological type prediction method based on twitter data and [9] mobile context aware acute stress prediction method are selected as comparison methods, and comparative experiments are designed from three aspects of prediction effect, prediction accuracy, and prediction efficiency.

4.2. Comparison of Predictive Effects of Psychological Pressure among College Students. In order to verify the prediction effect of the proposed method, the methods of [8] and the methods of [9] are compared with the proposed method respectively, and the comparison results of psychological pressure prediction effects of college students with different methods are obtained, as shown in Figure 7.

It can be seen from Figure 7 that under the sample data of different training sets, the prediction results of college students' psychological pressure in the method of [8] are large, which has a certain deviation from the actual prediction value, while the prediction results of college students' psychological pressure in the method of [9] are small, which has the largest deviation from the actual value. The prediction results of college students' psychological pressure by the proposed method are basically consistent with the actual prediction results of college students' psychological pressure. In the process of college students' psychological stress prediction, due to the influence of data personality, the fault tolerance of the prediction results of the two comparison methods is low, which reduces the prediction effect, while the deep neural network can reduce the influence of data to the minimum, improve the global training ability, and further optimize the prediction effect. Therefore, compared with the methods in [8] and [9], the proposed method has better prediction effect on college students' psychological stress.

\subsection{Comparison of Prediction Accuracy of Psychological} Pressure among College Students. On this basis, the prediction accuracy of the proposed method is further verified, and the ROC curve is used as the evaluation index of the prediction accuracy of the psychological pressure of college students. Among them, the AUC value is defined as the area under the ROC curve and the coordinate axis. Obviously, the value of this area will not be greater than 1 . The closer the AUC value is to 1 , the higher the authenticity of the prediction method of college students' psychological pressure, and the higher the prediction accuracy. By comparing the methods of [8], the methods of [9] and the proposed methods, we get the comparison results of the prediction accuracy of college students' psychological pressure by different methods, as shown in Figure 8.

It can be seen from Figure 8 that the AUC value of the method in [8] is 0.652, the AUC value of the method in [9] is 0.732 , and the AUC value of the proposed method is 0.856 . Therefore, compared with the method of [8] and the method of [9], the AUC value of the proposed method is larger and closer to 1, which shows that the college students'

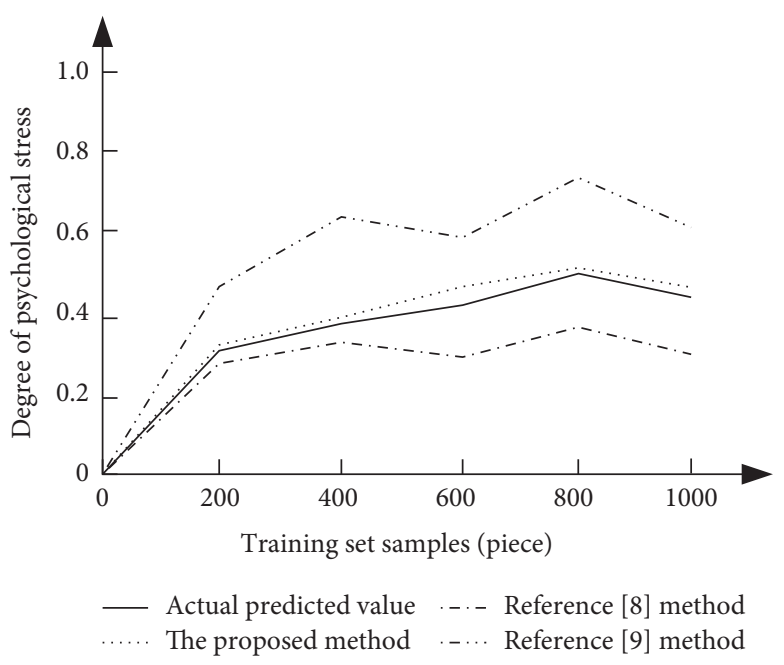

FIgURE 7: Comparison results of different methods of predicting the psychological pressure of college students.

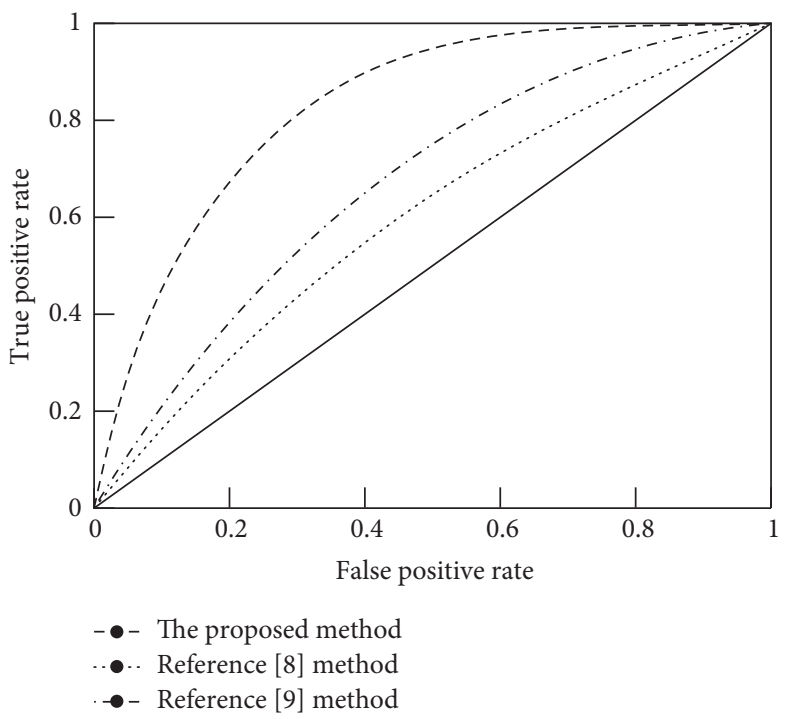

FIGURE 8: Comparison results of different methods of predicting the accuracy of college students' psychological pressure.

psychological stress prediction method based on deep neural network has higher authenticity and higher prediction accuracy. The main reason is that this method constructs the college students' psychological stress prediction model combined with the deep neural network method of gray theory, which improves the prediction accuracy.

4.4. Comparison of the Efficiency of Predicting Psychological Pressure among College Students. In order to further verify the prediction efficiency of the proposed method, the prediction time is taken as the evaluation index of the prediction efficiency of college students' psychological pressure. Among them, the shorter the prediction time, the higher the prediction efficiency of college students' psychological 


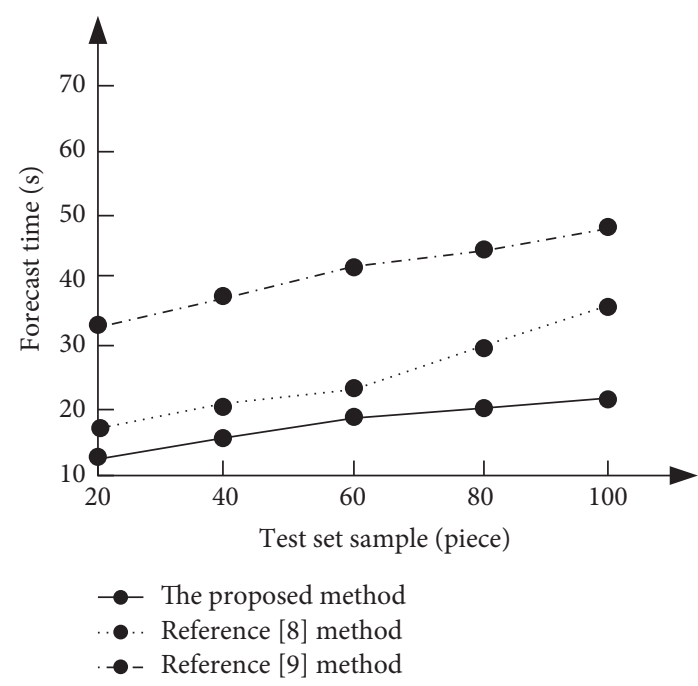

Figure 9: Comparison results of different methods of college students' psychological pressure prediction time.

pressure. By comparing the methods of [8], the methods of [9], and the proposed methods, the comparison results of college students' psychological pressure prediction time of different methods are obtained, as shown in Figure 9.

It can be seen from Figure 9 that with the increase of sample data in the test set, the prediction time of college students' psychological pressure with different methods increases. When the test set data is 100 , the prediction time of college students' psychological pressure by the method of [8] is $36 \mathrm{~s}$ and the prediction time of college students' psychological pressure by the method of [9] is $49 \mathrm{~s}$, while the prediction time of college students' psychological pressure by the proposed method is only $22 \mathrm{~s}$. It can be seen that, compared with the method of [8] and the method of [9], the proposed method has a short prediction time of college students' psychological stress, which can effectively improve the prediction efficiency of college students' psychological stress. The reason is that this method forms a physical network information model through the relationship between neurons, predicts the dynamic changes of college sudents' psychological stress, and improves the prediction efficiency of college students' psychological stress.

\section{Conclusion}

The prediction method of college students' psychological pressure based on deep neural network proposed in this paper gives full play to the advantages of deep neural network model and constructs the prediction model of college students' psychological pressure combined with gray theory model. The prediction accuracy and efficiency of college students' psychological pressure are high, and it has a good effect on psychological pressure prediction. However, in the process of college students' psychological pressure prediction, this method does not consider the influence of main influencing factors, such as different research groups and different research periods on college students' psychological pressure prediction results. Therefore, in the next research, it needs to fully consider the influence of groups in other periods, expand the number of tested samples, and make predictions in combination with their current situation, so as to further improve the reliability of prediction results.

\section{Data Availability}

The raw data supporting the conclusions of this article will be made available by the authors, without undue reservation.

\section{Conflicts of Interest}

The authors declare that they have no conflicts of interest regarding this work.

\section{References}

[1] D. Cortés-Denia, E. Lopez-Zafra, and M. Pulido-Martos, "Physical and psychological health relations to engagement and vigor at work: a PRISMA-compliant systematic review," Current Psychology, pp. 1-16, 2021.

[2] V. A. Trushin, A. I. Zavodovskaya, I. A. Oveshnikov, and E. V. Toporishchev, "Study of the impact of speech-like interference on the psychological and emotional state of a human," Journal of Physics: Conference Series, vol. 1791, no. 1, Article ID 012063, 2021.

[3] E. N. Sholokhova, E. V. Kazakova, and L. V. Sokolova, "Psychological component of school adaptation of firstgraders in the conditions of modern educational establishments of various types," ШсичолоГ, по. 5, pp. 33-44, 2020.

[4] B. W. Cowan and Z. Hao, "Medicaid expansion and the mental health of college students," Health Economics, vol. 30, no. 6, pp. 1306-1327, 2021.

[5] Y. H. Wang, Q. Y. Luo, and Z. T. Shi, "A study on the relationship between sexual morality education and mental health level of Chinese female college students," Medicine, vol. 98, no. 19, Article ID e15576, 2019.

[6] A. Fossas, "Psychological maturity predicts different forms of happiness," Journal of Happiness Studies, vol. 20, no. 6, pp. 1933-1952, 2019.

[7] M. Simbar, E. Zare, and Z. Shahhosseini, "Adolescent's psychological health in Iran," International Journal of Adolescent Medicine and Health, vol. 33, no. 4, pp. 221-228, 2019.

[8] A. Lima, L. D. Castro, and K. W. Tecla, "A temperament and psychological type prediction framework from Twitter data," PLoS One, vol. 14, no. 3, Article ID e0212844, 2019.

[9] R. Alharthi, R. Alharthi, B. Guthier, and A. El Saddik, "CASP: context-aware stress prediction system," Multimedia Tools and Applications, vol. 78, no. 7, pp. 9011-9031, 2019.

[10] M. Hauser, S. Gunn, S. Saab, and A. Ray, "State-space representations of deep neural networks," Neural Computation, vol. 31, no. 3, pp. 538-554, 2019.

[11] S. Herzog, C. Tetzlaff, and F. Wörgötter, "Evolving artificial neural networks with feedback," Neural Networks, vol. 123, pp. 153-162, 2020.

[12] T. Mandhula, S. Pabboju, and N. Gugulotu, "Predicting the customer's opinion on amazon products using selective memory architecture-based convolutional neural network," The Journal of Supercomputing, vol. 76, no. 8, pp. 5923-5947, 2020.

[13] Z.-k. Feng and W.-j. Niu, "Hybrid artificial neural network and cooperation search algorithm for nonlinear river flow 
time series forecasting in humid and semi-humid regions," Knowledge-Based Systems, vol. 211, p. 106580, 2021.

[14] C. Zhang, A. Liu, X. Liu, Y. Xu, and T. Li, "Interpreting and improving adversarial robustness of deep neural networks with neuron sensitivity," IEEE Transactions on Image Processing, vol. 30, pp. 1291-1304, 2020.

[15] Y. Ding, Z. Ma, S. Wen et al., "AP-CNN: weakly supervised attention pyramid convolutional neural network for finegrained visual classification," IEEE Transactions on Image Processing, vol. 30, pp. 2826-2836, 2021.

[16] K. Seddiki, P. Saudemont, F. Precioso et al., "Cumulative learning enables convolutional neural network representations for small mass spectrometry data classification," Nature Communications, vol. 11, no. 1, p. 5595, 2020.

[17] J. Yang, W. Fu, X. Cheng, X. Ye, and W. Zhao, "S2Engine: a novel systolic architecture for sparse convolutional neural networks," IEEE Transactions on Computers, vol. 15, no. 6, Article ID 3087946, 2021.

[18] L. Xu, L. Cui, T. Weise et al., "Semi-supervised multi-layer convolution kernel learning in credit evaluation," Pattern Recognition, vol. 120, no. 3, p. 108125, 2021.

[19] L. Ge, K. Wu, Y. Zeng, F. Chang, Y. Wang, and S. Li, "Multiscale spatiotemporal graph convolution network for air quality prediction," Applied Intelligence, vol. 51, no. 6, pp. 3491-3505, 2020.

[20] Chetna, P. Singh, and R. Kharab, "Coupled channel analysis of fusion excitation function for $9 \mathrm{Be}+64 \mathrm{Zn}$ system at near and above barrier energies," Modern Physics Letters A, vol. 35, no. 31, p. 2050257, 2020.

[21] M. Tripathy, S. R. Chiluveru, and B. Mohapatra, “An accuracy controlled iterative method for efficient sigmoid function approximation," Electronics Letters, vol. 56, no. 18, pp. 854$867,2020$.

[22] M. Cinar, I. Onder, A. Secer, T. A. Sulaiman, A. Yusuf, and M. Bayram, "Optical solitons of the $(2+1)$-dimensional Biswas-Milovic equation using modified extended tanh-function method," Optik, vol. 245, p. 167631, 2021.

[23] S. S. Husain and M. Bober, "REMAP: multi-layer entropyguided pooling of dense CNN features for image retrieval," IEEE Transactions on Image Processing, vol. 28, no. 10, pp. 5201-5213, 2019.

[24] A. Aghaee, P. Shamsipour, S. Hood, and R. Haugaard, "A convolutional neural network for semi-automated lineament detection and vectorisation of remote sensing data using probabilistic clustering: a method and a challenge," Computers \& Geosciences, vol. 151, p. 104724, 2021.

[25] S. Yang, J. Sun, Z. Yan, and X. Li, "Research on image interframe compensation based on deep convolutional neural network," Computer Simulation, vol. 37, no. 01, pp. 452-455, 2020.

[26] J. Luo and X. Zhang, "Convolutional neural network based on attention mechanism and Bi-LSTM for bearing remaining life prediction," Applied Intelligence, pp. 1-16, 2021.

[27] Q. Wang and F. Jiang, "Integrating linear and nonlinear forecasting techniques based on grey theory and artificial intelligence to forecast shale gas monthly production in Pennsylvania and Texas of the United States," Energy, vol. 178, pp. 781-803, 2019.

[28] W. Wu, X. Ma, Y. Zhang, W. Li, and Y. Wang, "A novel conformable fractional non-homogeneous grey model for forecasting carbon dioxide emissions of BRICS countries," The Science of the Total Environment, vol. 707, pp. 135447135447.24, 2020.
[29] W. Zhou, B. Zeng, J. Wang, X. Luo, and X. Liu, "Forecasting Chinese carbon emissions using a novel grey rolling prediction model," Chaos, Solitons \& Fractals, vol. 147, p. 110968 , 2021.

[30] M. M. Bejani and M. Ghatee, "Theory of adaptive SVD regularization for deep neural networks," Neural Networks, vol. 128, pp. 33-46, 2020.

[31] B. Zhao, Y. Ren, D. Gao, and L. Xu, "Performance ratio prediction of photovoltaic pumping system based on grey clustering and second curvelet neural network," Energy, vol. 171, pp. 360-371, 2019.

[32] B. Zhao, Y. Ren, D. Gao, L. Xu, and Y. Zhang, "Energy utilization efficiency evaluation model of refining unit Based on Contourlet neural network optimized by improved grey optimization algorithm," Energy, vol. 185, pp. 1032-1044, 2019.

[33] S. U. H. Dar, M. Özbey, A. B. Çatlı, and T. Çukur, “A transferlearning approach for accelerated MRI using deep neural networks," Magnetic Resonance in Medicine, vol. 84, no. 2, pp. $663-685,2020$.

[34] G. Kutyniok, P. Petersen, M. Raslan, and R. Schneider, "A theoretical analysis of deep neural networks and parametric PDEs," Constructive Approximation, vol. 304, Article ID s00365, 2021.

[35] J. Aghili and O. Mula, "Depth-adaptive neural networks from the optimal control viewpoint," ArXiv Preprint, vol. 5, no. 6, p. 02428, 2020.

[36] S. Langer, "Approximating smooth functions by deep neural networks with sigmoid activation function," Journal of Multivariate Analysis, vol. 182, Article ID 104696, 2021. 